PROCEEDINGS OF THE

AMERICAN MATHEMATICAL SOCIETY

Volume 134, Number 4, Pages 1087-1097

S 0002-9939(05)08055-X

Article electronically published on July 25, 2005

\title{
MAULDIN-WILLIAMS GRAPHS, MORITA EQUIVALENCE AND ISOMORPHISMS
}

\author{
MARIUS IONESCU
}

(Communicated by David R. Larson)

\begin{abstract}
We describe a method for associating some non-self-adjoint algebras to Mauldin-Williams graphs and we study the Morita equivalence and isomorphism of these algebras.

We also investigate the relationship between the Morita equivalence and isomorphism class of the $C^{*}$-correspondences associated with Mauldin-Williams graphs and the dynamical properties of the Mauldin-Williams graphs.
\end{abstract}

\section{Introduction}

In this note we follow the notation from [8]. By a Mauldin-Williams graph (see [14]), we mean a system $\mathcal{G}=\left(G,\left\{T_{v}, \rho_{v}\right\}_{v \in V},\left\{\phi_{e}\right\}_{e \in E}\right)$, where $G=(V, E, r, s)$ is a graph with a finite set of vertices $V$, a finite set of edges $E$, a range map $r$ and a source map $s$, and where $\left\{T_{v}, \rho_{v}\right\}_{v \in V}$ and $\left\{\phi_{e}\right\}_{e \in E}$ are families such that:

(1) Each $T_{v}$ is a compact metric space with a prescribed metric $\rho_{v}, v \in V$.

(2) For $e \in E, \phi_{e}$ is a continuous map from $T_{r(e)}$ to $T_{s(e)}$ such that

$$
c_{1} \rho_{r(e)}(x, y) \leq \rho_{s(e)}\left(\phi_{e}(x), \phi_{e}(y)\right) \leq c \rho_{r(e)}(x, y)
$$

for some constants $c_{1}, c$ satisfying $0<c_{1} \leq c<1$ (independent of $e$ ) and all $x, y \in T_{r(e)}$.

We shall assume, too, that the source map $s$ and the range map $r$ are surjective. Thus, we assume that there are no sinks and no sources in the graph $G$.

In [8] we associated to a Mauldin-Williams graph $\mathcal{G}=\left(G,\left\{T_{v}, \rho_{v}\right\}_{v \in V},\left\{\phi_{e}\right\}_{e \in E}\right)$ a so-called $C^{*}$-correspondence $\mathcal{X}$ over the $C^{*}$-algebra $A=C(T)$, where $T=$ $\coprod_{v \in V} T_{v}$ is the disjoint union of the spaces $T_{v}, v \in V$, as follows. Let $E \times_{G} T=$ $\left\{(e, x) \mid x \in T_{r(e)}\right\}$. Then, by our finiteness assumptions, $E \times{ }_{G} T$ is a compact space. We set $\mathcal{X}=C\left(E \times{ }_{G} T\right)$ and view $\mathcal{X}$ as a $C^{*}$-correspondence over $C(T)$ via the formulae:

$$
\begin{aligned}
& \xi \cdot a(e, x):=\xi(e, x) a(x), \\
& a \cdot \xi(e, x):=a \circ \phi_{e}(x) \xi(e, x)
\end{aligned}
$$

and

$$
\langle\xi, \eta\rangle_{A}(x):=\sum_{\substack{e \in E \\ x \in T_{r(e)}}} \overline{\xi(e, x)} \eta(e, x),
$$

Received by the editors September 1, 2004 and, in revised form, November 1, 2004.

2000 Mathematics Subject Classification. Primary 46K50, 46L08; Secondary 26A18, 37A55, 37B10, 37E25. 
where $a \in C(T)$ and $\xi, \eta \in C\left(E \times{ }_{G} T\right)$. With these data we can form the tensor algebra $\mathcal{T}_{+}(\mathcal{X})$ as prescribed in [15] and [16]. Our main result is:

Theorem 1.1. For $i=1,2$, let $\mathcal{G}_{i}=\left(G_{i},\left(K_{v}^{i}\right)_{v \in V_{i}},\left(\phi_{e}^{i}\right)_{e \in E_{i}}\right)$ be two MauldinWilliams graphs. Let $A_{i}=C\left(K^{i}\right)$ and let $\mathcal{X}_{i}$ be the associated $C^{*}$-algebras and $C^{*}$-correspondences. Then the following are equivalent:

(1) $\mathcal{T}_{+}\left(\mathcal{X}_{1}\right)$ is strongly Morita equivalent to $\mathcal{T}_{+}\left(\mathcal{X}_{2}\right)$ in the sense of 2 .

(2) $\mathcal{X}_{1}$ and $\mathcal{X}_{2}$ are strongly Morita equivalent in the sense of 16 .

(3) $\mathcal{X}_{1}$ and $\mathcal{X}_{2}$ are isomorphic as $C^{*}$-correspondeces.

(4) $\mathcal{T}_{+}\left(\mathcal{X}_{1}\right)$ is completely isometrically isomorphic to $\mathcal{T}_{+}\left(\mathcal{X}_{2}\right)$.

We find this result especially remarkable in light of Theorem 2.3 from [8, Theorem 1.1] (see also Section 4.2 from [18]), which states that the Cuntz-Pimsner algebra, $\mathcal{O}(\mathcal{X})$, which is the $C^{*}$-envelope of the tensor algebra $\mathcal{T}_{+}(\mathcal{X})$, depends only of the structure of the underlying graph. In particular, our results lead to examples of different non-self-adjoint algebras which are not completely isometrically isomorphic, but have the same $C^{*}$-envelope, namely $\mathcal{O}_{n}$.

To understand further the relationship between the tensor algebra and the Mauldin-Williams graph, we study the isomorphism class of our $C^{*}$-correspondences and tensor algebras in terms of the dynamics of the Mauldin-Williams graph. Roughly, we find that two $C^{*}$-correspondences associated to two Mauldin-Williams graphs, $\left(G_{i},\left(K_{v}^{(i)}\right)_{v \in V_{i}},\left(\phi_{e}^{i}\right)_{e \in E_{i}}\right), i=1,2$, are isomorphic if the maps $\phi_{e}^{1}$ and $\phi_{e}^{2}$ are locally conjugate in a sense that will be made precise later.

\section{NON-SELF-ADJOINT ALGEBRAS ASSOCIATED WITH MAULDIN-WILLIAMS GRAPHS}

Definition 2.1. An invariant list associated with a Mauldin-Williams graph $\mathcal{G}=$ $\left(G,\left\{T_{v}, \rho_{v}\right\}_{v \in V},\left\{\phi_{e}\right\}_{e \in E}\right\}$ is a family $\left(K_{v}\right)_{v \in V}$ of compact sets, such that $K_{v} \subset T_{v}$, for all $v \in V$ and such that

$$
K_{v}=\bigcup_{e \in E, s(e)=v} \phi_{e}\left(K_{r(e)}\right) .
$$

Since each $\phi_{e}$ is a proper contraction, $\mathcal{G}$ has a unique invariant list (see [14, Theorem 1]). We set $T:=\bigcup_{v \in V} T_{v}$ and $K:=\bigcup_{v \in V} K_{v}$ and we call $K$ the invariant set of the Mauldin-Williams graph.

In the particular case when we have one vertex $v$ and $n$ edges, i.e. in the setting of an iterated function system, the invariant set is the unique compact subset $K:=K_{v}$ of $T=T_{v}$ such that

$$
K=\phi_{1}(K) \cup \cdots \cup \phi_{n}(K) .
$$

Note that the $*$-homomorphism $\Phi: A \rightarrow \mathcal{L}(\mathcal{X}),(\Phi(a) \xi)(e, x)=a \circ \phi_{e}(x) \xi(e, x)$, which gives the left action of the $C^{*}$-correspondence associated to a MauldinWilliams graph, is faithful if and only if $K=T$. In this note we assume that $T$ equals the invariant set $K$.

Kajiwara and Watatani have proved in [10, Lemma 2.3] that, if the contractions are proper, the invariant set of an iterated function system has no isolated point. Their proof can be easily generalized to the invariant set of a Mauldin-Williams graph. Hence $K$ has no isolated points.

For a $C^{*}$-correspondence $\mathcal{X}$ over a $C^{*}$-algebra $A$, the (full) Fock space over $\mathcal{X}$ is

$$
\mathcal{F}(\mathcal{X})=A \oplus \mathcal{X} \oplus \mathcal{X}^{\otimes 2} \oplus \cdots
$$


We write $\Phi_{\infty}$ for the left action of $A$ on $\mathcal{F}(\mathcal{X}), \Phi_{\infty}(a)=\operatorname{diag}\left(a, \Phi^{(1)}(a), \Phi^{(2)}(a), \cdots\right)$, where $\Phi^{(n)}$ is the left action of $A$ on $\mathcal{X}^{\otimes n}\left(\Phi^{(1)}=\Phi\right.$, the left action of $A$ on $\left.\mathcal{X}\right)$. For $\xi \in \mathcal{X}$, the creation operator determined by $\xi$ is defined by the formula $T_{\xi}(\eta)=\xi \otimes \eta$, for all $\eta \in \mathcal{F}(\mathcal{X})$.

Definition 2.2. The tensor algebra of $\mathcal{X}$, denoted by $\mathcal{T}_{+}(\mathcal{X})$, is the norm closed subalgebra of $\mathcal{L}\left(\mathcal{F}(\mathcal{X})\right.$ ) generated by $\Phi_{\infty}(A)$ and the creation operators $T_{\xi}$, for $\xi \in \mathcal{X}$ (see [15] and [16]). The $C^{*}$-algebra generated by $\mathcal{T}_{+}(\mathcal{X})$ is denoted by $\mathcal{T}(\mathcal{X})$ and it is called the Toeplitz algebra of the $C^{*}$-correspondence $\mathcal{X}$.

We may regard each finite sum $\sum_{n=0}^{N} \mathcal{X} \otimes n$ as a subspace of $\mathcal{F}(\mathcal{X})$ and we may regard $\mathcal{L}\left(\sum_{n=0}^{N} \mathcal{X}^{\otimes n}\right)$ as a subalgebra of $\mathcal{L}(\mathcal{F}(\mathcal{X}))$ in the obvious way. Let $B$ be the $C^{*}$-subalgebra of $\mathcal{L}(\mathcal{F}(\mathcal{X}))$ generated by all the $\mathcal{L}\left(\sum_{n=0}^{N} \mathcal{X}^{\otimes n}\right)$ as $N$ ranges over the non-negative integers. Then $\mathcal{T}(\mathcal{X}) \subset M(B)$, the multiplier algebra of $B$. The Cuntz-Pimsner algebra $\mathcal{O}(\mathcal{X})$ is defined to be the image of $\mathcal{T}(\mathcal{X})$ in the corona algebra $M(B) / B$ (see [15 and 17]).

By a homomorphism from an $A_{1}-B_{1} C^{*}$-correspondence $\mathcal{X}_{1}$, to an $A_{2}-B_{2}$ $C^{*}$-correspondence $\mathcal{X}_{2}$ we mean a triple $(\alpha, V, \beta)$, where $\alpha: A_{1} \rightarrow A_{2}, \beta: B_{1} \rightarrow B_{2}$ are $C^{*}$-homomorphisms and $V: \mathcal{X}_{1} \rightarrow \mathcal{X}_{2}$ is a linear map such that $V(a \xi b)=$ $\alpha(a) V(\xi) \beta(b)$ and such that $\langle V(\xi), V(\eta)\rangle_{B_{2}}=\beta\left(\langle\xi, \eta\rangle_{B_{1}}\right)$ (see [16, Section 1]). When $A_{1}=A_{2}$ and $B_{1}=B_{2}$, we will consider $\alpha \in \operatorname{Aut}\left(A_{1}\right)$ and $\beta \in \operatorname{Aut}\left(B_{1}\right)$. This then forces $V$ to be isometric. If $V$ is also surjective, we shall say that $V$ is a correspondence isomorphism over $(\alpha, \beta)$. If, moreover, $A_{1}=B_{1}$ and $\alpha=\beta$, we say that $V$ is a correspondence isomorphism over $\alpha$.

A central concept for our work in this note is the strong Morita equivalence for $C^{*}$-correspondences defined in [16, Definition 2.1], which we review here.

Definition 2.3. If $\mathcal{X}$ is a $C^{*}$-correspondence over a $C^{*}$-algebra $A$, and $\mathcal{Y}$ is a $C^{*}$-correspondence over a $C^{*}$-algebra $B$, we say that $\mathcal{X}$ and $\mathcal{Y}$ are strongly Morita equivalent if $A$ and $B$ are strongly Morita equivalent via an $A$ - $B$ equivalence bimodule $\mathcal{Z}$ (in which case we write $A \stackrel{\mathrm{SME}}{\sim} \mathcal{Z} B$ ), for which there is an $A$ - $B$ correspondence isomorphism $(i d, W, i d)$ from $\mathcal{Z} \otimes_{B} \mathcal{Y}$ onto $\mathcal{X} \otimes_{A} \mathcal{Z}$. This means, in particular, that $W(a \xi b)=a W(\xi) b$ for all $a \in A, b \in B$ and $\xi \in \mathcal{Z} \otimes_{B} \mathcal{Y}$ and that $\langle W(\xi), W(\eta)\rangle_{B}=\langle\xi, \eta\rangle_{B}$

We say that a $C^{*}$-correspondence $\mathcal{X}$ over a $C^{*}$-algebra $A$ is aperiodic if: for all $n \geq 1$, for all $\xi \in \mathcal{X}^{\otimes n}$ and for all hereditary subalgebras $B \subseteq A$, we have

$$
\inf \left\{\left\|\Phi^{(n)}(a) \xi a\right\| \mid a \geq 0, a \in B,\|a\|=1\right\}=0 .
$$

It was proved in [16, Theorem 3.2, Theorem 3.5] that if $\mathcal{X}$ and $\mathcal{Y}$ are strongly Morita equivalent, then $\mathcal{T}_{+}(\mathcal{X})$ and $\mathcal{T}_{+}(\mathcal{Y})$ (respectively $\mathcal{T}(\mathcal{X})$ and $\mathcal{T}(\mathcal{Y}), \mathcal{O}(\mathcal{X})$ and $\mathcal{O}(\mathcal{Y})$ ) are strongly Morita equivalent. Also, if $\mathcal{X}$ and $\mathcal{Y}$ are aperiodic $C^{*}$-correspondences over the $C^{*}$-algebras $A$ and $B$, respectively, and if $\mathcal{T}_{+}(\mathcal{X})$ and $\mathcal{T}_{+}(\mathcal{Y})$ are strongly Morita equivalent in the sense of [2], then $\mathcal{X}$ and $\mathcal{Y}$ are strongly Morita equivalent (see [16, Theorem 7.2]).

To study the aperiodicity and strong Morita equivalence of $C^{*}$-correspondences associated to Mauldin-Williams graphs, we need the following lemma which gives an equivalent description of when a $C^{*}$-correspondence is aperiodic.

Lemma 2.4 ([16, Lemma 5.2]). The $C^{*}$-correspondence $\mathcal{X}$ is aperiodic if and only if given $a_{0} \in A, a_{0} \geq 0, \xi^{k} \in \mathcal{X}^{\otimes k}, 1 \leq k \leq n$, and $\varepsilon>0$, there is an $x$ in the 
hereditary subalgebra $\overline{a_{0} A a_{0}}$, with $x \geq 0$ and $\|x\|=1$, such that

$$
\left\|x a_{0} x\right\|>\left\|a_{0}\right\|-\varepsilon
$$

and

$$
\left\|\Phi^{(k)}(x) \xi^{k} x\right\|<\varepsilon \text { for } 1 \leq k \leq n .
$$

For a directed graph $G=(V, E, r, s)$ and for $k \geq 2$, we define

$$
E^{k}:=\left\{\alpha=\left(\alpha_{1}, \cdots, \alpha_{k}\right): \alpha_{i} \in E \text { and } r\left(\alpha_{i}\right)=s\left(\alpha_{i+1}\right), i=1, \cdots, k-1\right\}
$$

to be the set of paths of length $k$ in the graph $G$. We define also the infinite path space to be

$$
E^{\infty}:=\left\{\left(\alpha_{i}\right)_{i \in \mathbb{N}}: \alpha_{i} \in E \text { and } r\left(\alpha_{i}\right)=s\left(\alpha_{i+1}\right) \text { for all } i \in \mathbb{N}\right\} .
$$

For $\alpha \in E^{k}$, we write $\phi_{\alpha}=\phi_{\alpha_{1}} \circ \cdots \circ \phi_{\alpha_{k}}$.

Proposition 2.5. Let $\mathcal{G}=\left(G,\left(K_{v}\right)_{v \in V},\left(\phi_{e}\right)_{e \in E}\right)$ be a Mauldin-Williams graph with the invariant set $K$. Let $A=C(K)$ be the associated $C^{*}$-algebra and let $\mathcal{X}$ be the associated $C^{*}$-correspondence. Then the $C^{*}$-correspondence $\mathcal{X}$ is aperiodic.

Proof. Note that $\phi_{\alpha}: K_{r(\alpha)} \rightarrow K_{s(\alpha)}$, with $\alpha \in E^{k}$ and $k \in \mathbb{N}$, has a fixed point if and only if $r(\alpha)=s(\alpha)$, i.e. $\alpha$ is a cycle in the graph $G$.

Fix $n_{0} \in \mathbb{N}$, choose $k \in \mathbb{N}, 1 \leq k \leq n_{0}$; let $a_{0} \in A$ with $a_{0} \geq 0$; let $\xi^{k} \in \mathcal{X}^{\otimes k}$ and let $\varepsilon>0$. We verify the criterion in Lemma 2.4 first when $n_{0}=k=1$.

Without loss of generality, we assume that $\left\|a_{0}\right\|=1$. Then we can find $t_{0} \in K$ such that $\left|a_{0}\left(t_{0}\right)\right| \geq 1-\varepsilon$ and $t_{0}$ is not a fixed point for any $\phi_{e}, e \in E$. Let $v_{0} \in V$ be such that $t_{0} \in K_{v_{0}}$. Choose $\delta_{1}>0$ such that $B\left(t_{0}, \delta_{1}\right) \subset K_{v_{0}}$ and $B\left(\phi_{e}\left(t_{0}\right), \delta_{1}\right) \cap B\left(t_{0}, \delta_{1}\right)=\emptyset$ for all $e \in E$ for which $r(e)=v_{0}$. Let

$$
\delta_{2}:= \begin{cases}\min \left\{\rho_{v_{0}}\left(t_{0}, t\right) \mid a_{0}(t)=0\right\}, & \text { if }\left\{t \in K_{v_{0}}: a_{0}(t)=0\right\} \neq \emptyset, \\ \delta_{1}, & \text { otherwise. }\end{cases}
$$

Set $\delta=\min \left\{\delta_{1}, \delta_{2}\right\}$ and let $x \in A, x \geq 0$ be such that

$$
x(t)= \begin{cases}1, & \text { if } \quad t=t_{0}, \\ 0, & \text { if } \quad t \in K \backslash B\left(t_{0}, \delta\right) .\end{cases}
$$

Since $x(t)>0$ only when $a_{0}(t)>0$, it follows that $x \in \overline{a_{0} A a_{0}}$. Moreover, $x\left(t_{0}\right) a_{0}\left(t_{0}\right) x\left(t_{0}\right)>1-\varepsilon$, hence $\left\|x a_{0} x\right\|>1-\varepsilon$.

Fix $t \in K$. If $t \in B\left(t_{0}, \delta\right)$, then $\phi_{e}(t) \notin B\left(t_{0}, \delta\right)$, by our choice of $\delta_{1}$ and the fact that each map $\phi_{e}$ is a contraction, for all $e \in E$ such that $r(e)=v_{0}$; so $x \circ \phi_{e}(t) x(t)=0$. If $t \notin B\left(t_{0}, \delta\right)$, then $x(t)=0$, hence $x \circ \phi_{e}(t) x(t)=0$, for all $e \in E$ such that $t \in K_{r(e)}$. Therefore, $(\Phi(x) \xi x)(e, t)=x \circ \phi_{e}(t) \xi(e, t) x(t)=0$ for all $(e, t) \in E \times \times_{G} K$. Since

$$
\langle\Phi(x) \xi x, \Phi(x) \xi x\rangle_{A}(t)=\sum_{\substack{e \in E \\ t \in K_{r(e)}}}\left(x \circ \phi_{e}(t)\right)^{2}|\xi(e, t)|^{2} x(t)^{2},
$$

we see that $\|\Phi(x) \xi x\|=0$.

For $n_{0}=2$, we choose $t_{0} \in K$ such that $a_{0}\left(t_{0}\right)>1-\varepsilon$ and $t_{0}$ is not a fixed point for any $\phi_{\alpha}$ with $\alpha \in E^{2}$. Let $v_{0} \in V$ be such that $t_{0} \in K_{v_{0}}$. Let $\delta_{1}>0$ be such 
that $B\left(\phi_{\alpha}\left(t_{0}\right), \delta_{1}\right) \cap B\left(t_{0}, \delta_{1}\right)=\emptyset$, for all $\alpha \in E^{2}$ for which $r(\alpha)=v_{0}$, and such that $B\left(t_{0}, \delta_{1}\right) \subset K_{v_{0}}$. Choosing $\delta_{2}, \delta$ and $x$ as before, we conclude that $x \in \overline{a_{0} A a_{0}}$ and $\|x\|>1-\varepsilon$. Moreover, we have $x \circ \phi_{\alpha}(t) x(t)=0$ for all $t \in K, \alpha \in E \cup E^{2}$ (since $\phi_{\alpha}$ is a contraction, for all $\alpha \in E \cup E^{2}$ ); and since

$$
\begin{aligned}
& \left\langle\Phi^{(2)}(x) \xi^{2} x, \Phi^{(2)}(x) \xi^{2} x\right\rangle_{A}(t) \\
& \quad=\sum_{\substack{\alpha \in E^{2} \\
t \in K_{r(\alpha)}}}\left(x \circ \phi_{\alpha}(t)\right)^{2}\left|\xi_{2}^{2}\left(\alpha_{2}, t\right)\right|^{2}\left|\xi_{1}^{2}\left(\alpha_{1}, \phi_{\alpha_{1}}(t)\right)\right|^{2} x(t)^{2}=0,
\end{aligned}
$$

it follows that $\left\|\Phi^{(k)}(x) \xi^{k} x\right\|=0$ for $k=1,2$. Applying the same argument inductively, we see that $\mathcal{X}$ is an aperiodic $C^{*}$-correspondence.

Let $K^{1}$ and $K^{2}$ be two compact metric spaces. Let $A_{1}=C\left(K^{1}\right)$ and $A_{2}=$ $C\left(K^{2}\right)$. If $A_{1} \stackrel{\mathrm{SME}}{\sim}{ }_{\mathcal{Z}} A_{2}$, then the Rieffel correspondence determines a unique homeomorphism $f: K^{1} \rightarrow K^{2}$ and a unique Hermitian line bundle $\mathcal{L}$ over $\operatorname{Graph}(f)=$ $\left\{(x, f(x)): x \in K^{1}\right\}$, such that $\mathcal{Z}$ is isomorphic to $\Gamma(\mathcal{L})$ (see [21], 20, Section 3.3 and Example 4.55], [19, Appendix $(\mathrm{A})])$, where $\Gamma(\mathcal{L})$ is the imprimitivity bimodule of the cross sections of $\mathcal{L}$ endowed with the following structure:

$$
\begin{aligned}
(a \cdot s \cdot b)(x, f(x)) & =a(x) s(x, f(x)) b(f(x)), \\
\left\langle s_{1}, s_{2}\right\rangle_{A_{2}}(y) & \left.=\frac{s_{1}\left(f^{-1}(y), y\right)}{s_{2}\left(f^{-1}(y)\right.}, y\right), \\
A_{1}\left\langle s_{1}, s_{2}\right\rangle(x) & =s_{1}(x, f(x)) \overline{s_{2}(x, f(x))}
\end{aligned}
$$

for all $a \in A_{1}, b \in A_{2}, s, s_{1}, s_{2} \in \Gamma(\mathcal{L})$. We write $\mathcal{Z}(f, \mathcal{L})$ for $\Gamma(\mathcal{L})$.

We are ready to prove the main theorem.

Proof of Theorem 1.1. By Proposition 2.5, $\mathcal{X}_{1}$ and $\mathcal{X}_{2}$ are aperiodic $C^{*}$-correspondences. Using [16, Theorem 7.2], we obtain that (11) implies (2).

Now we show that (2) implies (3). Suppose that $\mathcal{X}_{1}$ and $\mathcal{X}_{2}$ are strongly Morita equivalent. This implies that $A_{1}$ and $A_{2}$ are strongly Morita equivalent via an imprimitivity bimodule $\mathcal{Z}$ such that $\mathcal{Z} \otimes \mathcal{X}_{2}$ is isomorphic to $\mathcal{X}_{1} \otimes \mathcal{Z}$. Let $f: K^{1} \rightarrow$ $K^{2}$ and $\mathcal{L}$ be the homeomorphism and the line bundle determined by the Rieffel correspondence. We have that $\mathcal{Z}(f, \mathcal{L}) \otimes \mathcal{X}_{2}$ is isomorphic to $\mathcal{X}_{1} \otimes \mathcal{Z}(f, \mathcal{L})$. Hence $\mathcal{Z}(f, \mathcal{L}) \otimes \mathcal{X}_{2} \otimes \widetilde{\mathcal{Z}(f, \mathcal{L})}$ is isomorphic to $\mathcal{X}_{1}$, where $\widetilde{\mathcal{Z}(f, \mathcal{L})}$ is the dual imprimitivity bimodule (see [20, Proposition 3.18]). We prove that $\mathcal{Z}(f, \mathcal{L}) \otimes \mathcal{X}_{2} \otimes \widetilde{\mathcal{Z}(f, \mathcal{L})}$ is isomorphic to $\mathcal{X}_{2}$ over an isomorphism $\alpha$ of $A_{1}$ and $A_{2}$.

Let $\alpha: A_{1} \rightarrow A_{2}$ be defined by the formula $\alpha(a)=a \circ f^{-1}$ and let $V: \mathcal{Z}(f, \mathcal{L}) \otimes$ $\mathcal{X}_{2} \otimes \widetilde{\mathcal{Z}(f, \mathcal{L})} \rightarrow \mathcal{X}_{2}$ be defined by the formula

$$
V\left(s_{1} \otimes \xi \otimes \widetilde{s_{2}}\right)(e, y)=s_{1}\left(f^{-1}\left(\phi_{e}^{2}(y)\right), \phi_{e}^{2}(y)\right) \xi(e, x) \overline{s_{2}\left(f^{-1}(y), y\right)} .
$$

Then $\alpha$ is an isomorphism and

$$
V\left(a \cdot s_{1} \otimes \xi \otimes \widetilde{s_{2}} \cdot b\right)=a \cdot V\left(s_{1} \otimes \xi \otimes \widetilde{s_{2}}\right) \cdot b,
$$


for all $a, b \in A, s_{1}, s_{2} \in \mathcal{Z}(f, \mathcal{L}), \xi \in \mathcal{X}_{2}$. Moreover, we have that

$$
\begin{aligned}
& \left\langle V\left(s_{1} \otimes \xi \otimes \widetilde{s_{2}}\right), V\left(t_{1} \otimes \eta \otimes \widetilde{t_{2}}\right\rangle_{A_{2}}(y)\right. \\
& =\sum_{\substack{e \in E \\
y \in K_{r(e)}^{2}}} \overline{V\left(s_{1} \otimes \xi \otimes \widetilde{s_{2}}\right)}(e, y) V\left(\overline{\left.t_{1} \otimes \eta \otimes \widetilde{t_{2}}\right)(e, y)}\right. \\
& =\sum_{\substack{e \in E \\
y \in K_{r(e)}^{2}}}\left(\overline{s_{1}\left(f^{-1}\left(\phi_{e}^{2}(y)\right), \phi_{e}^{2}(y)\right) \xi(e, x) \overline{s_{2}\left(f^{-1}(y), y\right)}}\right. \\
& \left.\cdot t_{1}\left(f^{-1}\left(\phi_{e}^{2}(y)\right), \phi_{e}^{2}(y)\right) \eta(e, x) \overline{t_{2}\left(f^{-1}(y), y\right)}\right) \\
& =\left\langle s_{1} \otimes \xi \otimes s_{2}, t_{1} \otimes \eta \otimes t_{2}\right\rangle_{A_{2}},
\end{aligned}
$$

for all $s_{1}, s_{2}, t_{1}, t_{2} \in \mathcal{Z}(f, \mathcal{L})$ and $\xi, \eta \in \mathcal{X}_{2}$. Also, for $\xi \in \mathcal{X}_{2}, V(1 \otimes \xi \otimes 1)=\xi$. Hence $V$ is a correspondence isomorphism. Thus $\mathcal{X}_{1}$ is isomorphic to $\mathcal{X}_{2}$.

The rest is clear.

It was shown in [8, Theorem 2.3] that the Cuntz-Pimsner algebra of the $C^{*}$ correspondence built from a Mauldin-Williams graph is isomorphic to the CuntzKrieger algebra of the underlying graph $G=(V, E, r, s)$ (as defined in [12]). Hence, for $C^{*}$-correspondences associated to Mauldin-Williams graphs with the same underlying graph which are not isomorphic, we obtain tensor algebras which are not Morita equivalent, but have the same $C^{*}$-envelope, namely the Cuntz-Krieger algebra of the graph $G$.

\section{The ISOMORPHisM CLASS OF THE $C^{*}$-CORRESPONDENCES ASSOCIATED WITH MAULDIN-WILLIAMS GRAPHS}

In the following we analyze the relation between the isomorphism class of the $C^{*}$ correspondences associated with two Mauldin-Williams graphs, $\mathcal{G}_{i}=\left(G,\left(K_{v}^{i}\right)_{v \in V}\right.$, $\left.\left(\phi_{e}^{i}\right)_{e \in E}\right), i=1,2$, and the topological and dynamical properties of the MauldinWilliams graphs.

Since, by [18, Section 4.2] and 8, Theorem 2.3], the Cuntz-Pimsner algebra associated to a Mauldin-Williams graph depends only on the structure of the underlying graph $G$, we will consider only Mauldin-Williams graphs having the same underlying graph $G=(V, E, r, s)$.

Next we determine necessary and sufficient conditions for the isomorphism of the $C^{*}$-correspondences associated to two Mauldin-Williams graphs.

Proposition 3.1. For $i=1,2$, let $\mathcal{G}_{i}=\left(G,\left(K_{v}^{i}\right)_{v \in V},\left(\phi_{e}^{i}\right)_{e \in E}\right)$ be two MauldinWilliams graphs over the same underlying graph $G$. Let $A_{i}=C\left(K^{i}\right), i=1,2$, be the associated $C^{*}$-algebras and let $\mathcal{X}_{i}, i=1,2$, be the associated $C^{*}$-correspondences. If there is a homeomorphism $f: K^{1} \rightarrow K^{2}$, a partition of open subsets $\left\{U_{1}, \ldots, U_{m}\right\}$ for $K^{1}$, for some $m \in \mathbb{N}$, and if for each $U_{j}$ there is a permutation $\sigma_{j} \in S_{n}$, where $n=|E|$, such that $\left.f^{-1} \circ \phi_{\sigma_{j}(e)}^{2} \circ f\right|_{U_{j}}=\left.\phi_{e}^{1}\right|_{U_{j}}$ and $f\left(K_{r(e)}^{1}\right)=K_{r\left(\sigma_{j}(e)\right)}^{2}$ for all $e \in E$, $j \in\{1, \ldots, m\}$, then $\mathcal{X}_{1}$ and $\mathcal{X}_{2}$ are isomorphic.

Proof. Since $f$ is a homeomorphism, the map $\beta: A_{2} \rightarrow A_{1}$, defined by the equation $\beta(b)=b \circ f$ for all $b \in A_{2}$, is a $C^{*}$-isomorphism. Define $V: \mathcal{X}_{2} \rightarrow \mathcal{X}_{1}$ by the formula

$$
V(\xi)(e, x)=\sum_{k=1}^{m} \xi_{\sigma_{k}(e)}(f(x)) \cdot 1_{U_{k}}(x),
$$


for all $(e, x) \in E \times_{G} K$, where $\xi_{\sigma_{k}(e)}(f(x)):=\xi\left(\sigma_{k}(e), f(x)\right)$. We show that $V$ is a $C^{*}$-correspondence isomorphism over $\beta$. Let $b_{1}, b_{2} \in A_{2}$ and $\xi \in \mathcal{X}_{2}$. We have

$$
\begin{aligned}
V\left(b_{1} \cdot \xi \cdot b_{2}\right)(e, x) & =\sum_{k=1}^{m} b_{1} \circ \phi_{\sigma_{k}(e)}^{2}(f(x)) \xi_{\sigma_{k}(e)}(f(x)) b_{2}(f(x)) 1_{U_{k}}(x) \\
& =\sum_{k=1}^{m} b_{1} \circ f \circ \phi_{e}^{1}(x) \xi_{\sigma_{k}(e)}(f(x)) 1_{U_{k}}(x) \cdot \beta\left(b_{2}\right)(x) \\
& =\beta\left(b_{1}\right) \cdot V(\xi) \cdot \beta\left(b_{2}\right)(e, x) .
\end{aligned}
$$

Also

$$
\langle V(\xi), V(\eta)\rangle_{A_{1}}(x)=\sum_{\substack{e \in E \\ f(x) \in K_{r(e)}^{2}}}\left(\sum_{k=1}^{m} \overline{\xi_{\sigma_{k}(e)}(f(x))} \eta_{\sigma_{k}(e)}(f(x)) 1_{U_{k}}(x)\right),
$$

hence $\langle V(\xi), V(\eta)\rangle_{A_{1}}=\beta\left(\langle\xi, \eta\rangle_{A_{2}}\right)$. Finally, one can see that $V$ is onto, hence $V$ is a $C^{*}$-correspondence isomorphism.

Recall that, for $k \geq 2, E^{k}:=\left\{\alpha=\left(\alpha_{1}, \cdots, \alpha_{k}\right) \quad: \quad \alpha_{i} \in E\right.$ and $r\left(\alpha_{i}\right)=$ $\left.s\left(\alpha_{i+1}\right), i=1, \cdots, k-1\right\}$, is the set of paths of length $k$ in the graph $G$. Let $E^{*}=\bigcup_{k \in N} E^{k}$ be the space of finite paths in the graph $G$. Also the infinite path space, $E^{\infty}$, is defined to be

$$
E^{\infty}:=\left\{\left(\alpha_{i}\right)_{i \in \mathbb{N}} \quad: \quad \alpha_{i} \in E \quad \text { and } \quad r\left(\alpha_{i}\right)=s\left(\alpha_{i+1}\right) \text { for all } i \in \mathbb{N}\right\} .
$$

For $v \in V$, we also define $E^{k}(v):=\left\{\alpha \in E^{k}: s(\alpha)=v\right\}$, and $E^{*}(v)$ and $E^{\infty}(v)$ are defined similarly. We consider $E^{\infty}(v)$ to be endowed with the metric: $\delta_{v}(\alpha, \beta)=c^{|\alpha \wedge \beta|}$ if $\alpha \neq \beta$ and 0 otherwise, where $\alpha \wedge \beta$ is the longest common prefix of $\alpha$ and $\beta$, and $|w|$ is the length of the word $w \in E^{*}$ (see [5, Page 116]). Then $E^{\infty}(v)$ is a compact metric space, and, since $E^{\infty}$ equals the disjoint union of the spaces $E^{\infty}(v), E^{\infty}$ becomes a compact metric space in a natural way. Define the maps $\theta_{e}$ : $E^{\infty}(r(e)) \rightarrow E^{\infty}(s(e))$ by the formula $\theta_{e}(\alpha)=e \alpha$, for all $\alpha \in E^{\infty}$ and for all $e \in$ $E$. Then $\left(G,\left(E^{\infty}(v)\right)_{v \in V},\left(\theta_{e}\right)_{e \in E}\right)$ is a Mauldin-Williams graph. We set $A_{E}:=$ $C\left(E^{\infty}\right)$ and we set $\mathcal{E}$ be the $C^{*}$-correspondence associated to this Mauldin-Williams graph. Let $\mathcal{M}=\left(G,\left\{K_{v}, \rho_{v}\right\}_{v \in E^{0}},\left\{\phi_{e}\right\}_{e \in E^{1}}\right)$ be a Mauldin-Williams graph. For $\left(\alpha_{1}, \ldots, \alpha_{n}\right) \in E^{n}$ let $K_{\left(\alpha_{1}, \ldots, \alpha_{n}\right)}:=\phi_{\alpha_{1}} \circ \ldots \phi_{\alpha_{n}}\left(K_{r\left(\alpha_{n}\right)}\right)$. Then, for any infinite path $\alpha=\left(\alpha_{n}\right)_{n \in \mathbb{N}} \in E^{\infty}, \bigcap_{n \geq 1} K_{\left(\alpha_{1}, \ldots, \alpha_{n}\right)}$ contains only one point. Therefore, we can define a map $\pi: E^{\infty} \rightarrow K$ by $\{\pi(x)\}=\bigcap_{n>1} K_{\left(\alpha_{1}, \ldots, \alpha_{n}\right)}$. Since $\pi\left(E^{\infty}\right)$ is also an invariant set, $\pi$ is a continuous, onto map and $\pi\left(E^{\infty}(v)\right)=K_{v}$. Moreover, $\pi \circ \theta_{e}=\phi_{e} \circ \pi$.

We say that a Mauldin-Williams graph $\mathcal{M}=\left(G,\left\{K_{v}, \rho_{v}\right\}_{v \in E^{0}},\left\{\phi_{e}\right\}_{e \in E^{1}}\right)$ is totally disconnected if $\phi_{e}\left(K_{r(e)}\right) \cap \phi_{f}\left(K_{r(f)}\right)=\emptyset$ if $s(e)=s(f)$ and $e \neq f$.

Corollary 3.2. Let $\mathcal{M}=\left(G,\left\{K_{v}, \rho_{v}\right\}_{v \in E^{0}},\left\{\phi_{e}\right\}_{e \in E^{1}}\right)$ be a totally disconnected Mauldin-Williams graph. Let $A$ be the $C^{*}$-algebra and let $\mathcal{X}$ be the $C^{*}$-correspondence associated to this Mauldin-Williams graph. Then $\mathcal{X}$ is isomorphic with $\mathcal{E}$, as $C^{*}$-correspondences. In particular, one obtains that for any two totally disconnected Mauldin-Williams graphs having the same underlying graph $G$, the $C^{*}$ correspondences and tensor algebras associated are isomorphic. 
Proof. If the Mauldin-Williams graph is totally disconnected, then the map $\pi$ : $E^{\infty} \rightarrow K$ defined above is a homeomorphism. Moreover, $\pi \circ \theta_{e} \circ \pi^{-1}=\phi_{e}$ for all $e \in E$, therefore the associated $C^{*}$-correspondences are isomorphic.

The converse of this corollary is true and will be proved later.

The next theorem is a converse of the Proposition 3.1. We note, however, that the family of open sets $\left\{U_{i}\right\}$ here is not required to be a partition of the compact set $K^{1}$, but only a finite open cover of it.

Theorem 3.3. For $i=1,2$, let $\mathcal{G}_{i}=\left(G,\left(K_{v}^{i}\right)_{v \in V},\left(\phi_{e}^{i}\right)_{e \in E}\right)$ be two MauldinWilliams graphs over the same underlying graph $G$. Let $A_{i}=C\left(K^{i}\right), i=1,2$, be the associated $C^{*}$-algebras and let $\mathcal{X}_{i}, i=1,2$, be the associated $C^{*}$-correspondences. If $\mathcal{X}_{1}$ and $\mathcal{X}_{2}$ are isomorphic, then there is a homeomorphism $f: K^{1} \rightarrow K^{2}$, a finite open cover of $K^{1},\left\{U_{1}, \ldots, U_{m}\right\}$, and for each $U_{j}$ there is a permutation $\sigma_{j} \in S_{n}$ $(n=|E|)$ such that

$$
\left.f^{-1} \circ \phi_{e}^{2} \circ f\right|_{U_{j}}=\left.\phi_{\sigma_{j}(e)}^{1}\right|_{U_{j}} \text { for all } e \in E, i \in\{1, \ldots, m\} .
$$

Proof. Since $\mathcal{X}_{1}$ and $\mathcal{X}_{2}$ are isomorphic, there is a $C^{*}$-isomorphism $\beta: A_{2} \rightarrow A_{1}$ and a $C^{*}$-correspondence isomorphism $W: \mathcal{X}_{2} \rightarrow \mathcal{X}_{1}$ such that $W\left(b_{1} \cdot \xi \cdot b_{2}\right)=$ $\beta\left(b_{1}\right) W(\xi) \beta\left(b_{2}\right)$ and $\langle W(\xi), W(\eta)\rangle_{A_{1}}=\beta\left(\langle\xi, \eta\rangle_{A_{2}}\right)$, for all $b_{1}, b_{2} \in A_{2}, \xi, \eta \in \mathcal{X}_{2}$. Let $f: K^{1} \rightarrow K^{2}$ be the homeomorphism which implements $\beta$, that is, $\beta(b)=b \circ f$, for all $b \in A_{2}$.

Let $\delta_{e} \in \mathcal{X}_{2}$, defined by

$$
\delta_{e}(g, y)=\left\{\begin{array}{lc}
1, & \text { if } e=g \\
0, & \text { otherwise }
\end{array}\right.
$$

for $e \in E$, be the natural basis in $\mathcal{X}_{2}$ and let $\left(\delta_{e}^{\prime}\right)_{e \in E} \subset \mathcal{X}_{1}$ be the natural basis in $\mathcal{X}_{1}$, which is defined similarly.

For $\xi \in \mathcal{X}_{2}, \xi=\sum_{g \in E} \delta_{g} \cdot \xi_{g}$, where $\xi_{g}(y)=\xi(g, y)$ for all $y \in K_{r(g)}^{2}$ and is 0 otherwise. With respect to the bases, we can write

$$
\begin{aligned}
W(\xi) & =W\left(\sum_{g \in E} \delta_{g} \cdot \xi_{g}\right)=\sum_{g \in E} W\left(\delta_{g}\right) \cdot \xi_{g} \circ f \\
& =\sum_{g \in E} \sum_{e \in E} \delta_{e}^{\prime} \cdot w_{e g} \xi_{g} \circ f
\end{aligned}
$$

where

$$
W\left(\delta_{g}\right)=\sum_{e \in E} \delta_{e}^{\prime} \cdot w_{e g}, \quad w_{e g} \in A_{1},
$$

and $w_{e g}$ are given by the formula $w_{e g}=\left\langle\delta_{e}^{\prime}, W\left(\delta_{g}\right)\right\rangle_{A_{1}}$, for all $e, g \in E$. We call $\left(w_{e g}\right)_{e, g \in E}$ the matrix of $W$ with respect to the basis $\left(\delta_{e}^{\prime}\right)_{e \in E}$ and $\left(\delta_{g}\right)_{g \in E}$ (it is an $n \times n$ matrix, where $n=|E|)$. Since $W$ preserves the inner product, we see that

$$
\left\langle W\left(\delta_{g}\right), W\left(\delta_{e}\right)\right\rangle=\left\langle\delta_{g}, \delta_{e}\right\rangle=\delta_{g e},
$$

where $\delta_{g e}(x)=1$ if $e=g$ and $x \in K_{r(e)}$ and is 0 otherwise. Also,

$$
\left\langle W\left(\delta_{g}\right), W\left(\delta_{e}\right)\right\rangle=\sum_{f \in E} w_{f g}^{*} w_{f e} .
$$


Equations (3.4) and (3.5) imply that for every $x \in K^{1}$ the matrix $\left(w_{e f}(x)\right)_{e, f \in E}$ is invertible. Hence there is $\sigma_{x} \in S_{n}$ such that $w_{\sigma_{x}(e) e}(x) \neq 0$ for all $e \in E$. Therefore, there is a neighborhood $U_{x}$ of $x$ such that

$$
w_{\sigma_{x}(e) e}(y) \neq 0 \text { for all } e \in E, y \in U_{x} \text { and } x \in K^{1} .
$$

Let $b \in A_{2}$. Then, for $h \in E$ we have that

$$
W\left(b \cdot \delta_{h}\right)=\sum_{e \in E} \delta_{e}^{\prime} w_{e h} b \circ \phi_{h}^{2} \circ f
$$

and

$$
\beta(b) \cdot W\left(\delta_{h}\right)=\sum_{e \in E} \delta_{e}^{\prime} b \circ f \circ \phi_{e}^{1} w_{e h} .
$$

Fix $x \in K^{1}$ and let $\sigma_{x} \in S_{n}$ and $U_{x}$ be defined as in Equation (3.6). Then

$$
W\left(b \cdot \delta_{h}\right)\left(\sigma_{x}(h), y\right)=w_{\sigma_{x}(h) h}(y) b \circ \phi_{h}^{2} \circ f(y)
$$

and

$$
\left(\beta(b) \cdot W\left(\delta_{h}\right)\right)\left(\sigma_{x}(h), y\right)=b \circ f \circ \phi_{\sigma_{x}(h)}^{1}(y) w_{\sigma_{x}(h) h}(y)
$$

for all $y \in U_{x}$ and for all $h \in E$. Since $W$ is a $C^{*}$-correspondence isomorphism and $w_{\sigma_{x}(h) h}(y) \neq 0$ for all $y \in U_{x}$, for any $x \in K^{1}$, there is a neighborhood $U_{x}$ of $x$ in $K^{1}$ and there is a permutation $\sigma_{x} \in S_{n}$ such that

$$
\left.f^{-1} \circ \phi_{h}^{2} \circ f\right|_{U_{x}}=\left.\phi_{\sigma_{x}(h)}^{1}\right|_{U_{x}} \text { for all } h \in E .
$$

Hence we can find a finite cover $\left\{U_{1}, \ldots, U_{m}\right\}$ of $K^{1}$ and for each $U_{i}$ we can find a permutation $\sigma_{i} \in S_{n}$ such that the Equation (3.1) holds.

In the special case when the two Mauldin-Williams graphs are totally disconnected, more can be said about the choice of the permutations $\sigma_{i}$.

Corollary 3.4. Let $\mathcal{G}_{i}=\left(G,\left(K_{v}^{i}\right)_{v \in V},\left(\phi_{e}^{i}\right)_{e \in E}\right)$ be two Mauldin-Williams graphs. Let $A_{i}=C\left(K^{i}\right)$ and let $\mathcal{X}_{i}, i=1,2$, be the associated $C^{*}$-algebras and $C^{*}$ correspondences. If $\mathcal{G}_{1}$ is totally disconnected and if $\mathcal{X}_{1}$ is isomorphic with $\mathcal{X}_{2}$ there is a continuous map $h: K^{1} \rightarrow S_{n}$ such that $f^{-1} \circ \phi_{e}^{2} \circ f(x)=\phi_{h(x)(e)}(x)$, for all $x \in$ $K^{1}$.

Proof. Recall that if $\mathcal{G}_{1}$ is totally disconnected, then $\phi_{e}^{1}\left(K_{r(e)}\right) \cap \phi_{f}^{1}\left(K_{r(f)}\right)=\emptyset$ if $e \neq f$. From the Theorem 3.3 we know that there are open sets $\left\{U_{1}, \cdots, U_{m}\right\}$, for some $m \in \mathbb{N}$, and permutations $\sigma_{1}, \cdots, \sigma_{m} \in S_{n}$ such that

$$
\left.f^{-1} \circ \phi_{e}^{2} \circ f\right|_{U_{i}}=\left.\phi_{\sigma_{i}(e)}^{1}\right|_{U_{i}} \quad \text { for all } e \in E, i \in\{1, \cdots, m\} .
$$

If $U_{i} \cap U_{j} \neq \emptyset$ for some $i \neq j$, then it follows that $\phi_{\sigma_{i}(e) \mid U_{i} \cap U_{j}}^{1}=\left.\phi_{\sigma_{j}(e)}^{1}\right|_{U_{i} \cap U_{j}}$ for all $e \in E$, hence $\sigma_{i}(e)=\sigma_{j}(e)$ for all $e \in E$, so $\sigma_{i}=\sigma_{j}$. Therefore, we can choose the cover $U_{1}, \cdots, U_{m}$ such that $U_{i} \cap U_{j}=\emptyset$ if $i \neq j$.

Let $x \in K^{1}$. Then there is a unique $i \in\{1, \cdots, n\}$ such that $x \in U_{i}$. We define $h(x)=\sigma_{i}$. Then $h: K^{1} \rightarrow S_{n}$ is a well-defined map. Moreover, $h$ is continuous (considering $S_{n}$ endowed with the discrete topology), since for every $\sigma \in S_{n}, h^{-1}(\sigma)=\emptyset$ or $h^{-1}(\sigma)=U_{i}$, for some $i \in\{1, \cdots, n\}$. Finally, from the Equation (3.7) we obtain that

$$
f^{-1} \circ \phi_{e}^{2} \circ f(x)=\phi_{h(x)(e)}^{1}(x) \text { for all } x \in K^{1} \text { and } e \in E .
$$


Suppose that $\mathcal{G}_{i}=\left(G,\left(K_{v}^{i}\right)_{v \in V},\left(\phi_{e}^{i}\right)_{e \in E}\right)$ are two Mauldin-Williams graphs that satisfy the hypothesis of the Corollary 3.4. We claim that $\mathcal{G}_{2}$ must also be totally disconnected. Suppose that there are $e, f \in E, e \neq f$, such that $\phi_{e}^{2}\left(K_{r(e)}^{2}\right) \cap$ $\phi_{f}^{2}\left(K_{r(f)}^{2}\right) \neq \emptyset$. Then there is an $x \in K^{1}$ such that $y:=f(x) \in \phi_{e}^{2}\left(K_{r(e)}^{2}\right) \cap$

$\phi_{f}^{2}\left(K_{r(f)}^{2}\right)$. Then $\phi_{h(x)(e)}^{1}(x)=\phi_{h(x)(f)}^{1}(x)$, which is a contradiction since $\mathcal{G}_{1}$ is totally disconnected. So $\mathcal{G}_{2}$ is totally disconnected.

Example 3.5. Let $K$ be the Cantor set, let $\phi_{i}: K \rightarrow K, i=1,2$, be the maps defined by the formulae $\phi_{1}(x)=\frac{1}{3} x$ and $\phi_{2}(x)=\frac{1}{3} x+\frac{2}{3}$. Then $K$ is the invariant set of $\left(\phi_{1}, \phi_{2}\right)$. Let $T=[0,1]$ and let $\psi_{i}: T \rightarrow T, i=1,2$, be the maps defined by the formulae $\psi_{1}(x)=\frac{1}{2} x$ and $\psi_{2}(x)=-\frac{1}{2} x+1$. Then $T$ is the invariant set of $\left(\psi_{1}, \psi_{2}\right)$. Since $\left(\psi_{1}, \psi_{2}\right)$ is not totally disconnected, we see that the associated $C^{*}$ correspondences are not strongly Morita equivalent. Hence the tensor algebras fail to be strongly Morita equivalent in the sense of [2], yet their $C^{*}$-envelopes coincide with $\mathcal{O}_{2}$.

Example 3.6. Let $T$ be the regular triangle in $\mathbb{R}^{2}$ with vertices $A=(0,0), B=$ $(1,0)$ and $C=(1 / 2, \sqrt{3} / 2)$. Let $\phi_{1}(x, y)=\left(\frac{x}{2}+\frac{1}{4}, \frac{y}{2}+\frac{\sqrt{3}}{4}\right), \phi_{2}(x, y)=\left(\frac{x}{2}, \frac{y}{2}\right)$ and $\phi_{3}(x, y)=\left(\frac{x}{2}+\frac{1}{2}, \frac{y}{2}\right)$. Then the invariant set $K$ of this iterated function system is the Sierpinski gasket. Let $\psi_{1}=\sigma_{1} \circ \phi_{1}, \psi_{2}=\phi_{2}$ and $\psi_{3}=\sigma_{3} \circ \phi_{3}$, where $\sigma_{i}$ is the symmetry about the median from the vertex $\phi_{i}(C)$ of the triangle $\phi_{i}(T)$, for $i=1,3$. Then the invariant set of this iterated function system is also the Sierpinski gasket, but the $C^{*}$-correspondences associated to $\left(\phi_{1}, \phi_{2}, \phi_{3}\right)$ and $\left(\psi_{1}, \psi_{2}, \psi_{3}\right)$ are not isomorphic.

\section{REFERENCES}

[1] M. F. Barnsley, Fractals everywhere, Second edition, Academic Press Professional, Boston, MA, 1993. MR1231795 (94h:58101)

[2] D. P. Blecher, P. S. Muhly, V. I. Paulsen, 'Categories of operator modules (Morita equivalence and projective modules).' Mem. Amer. Math. Soc. 143 (2000), no. 681, viii+94 pp. MR.1645699 (2000j:46132)

[3] J. Cuntz, 'Simple $C^{*}$-algebras generated by isometries', Comm. Math. Phys, 57 (1977), no. 2, 173-185. MR0467330 (57:7189)

[4] J. Cuntz and W. Krieger, 'A class of $C^{*}$-algebras and topological Markov chains', Invent. Math. 56 (1980), no. 3, 251-268. MR0561974 (82f:46073a)

[5] G. A. Edgar, Measure, topology, and fractal geometry, Undergraduate Texts in Mathematics. Springer-Verlag, New York, 1990. MR.1065392 (92a:54001)

[6] N. J. Fowler, P. S. Muhly and I. Raeburn, 'Representations of Cuntz-Pimsner Algebras', Indiana Univ. Math. J. 52 (2003), no. 3, 569-605. MR.1986889

[7] J. E. Hutchinson, 'Fractals and self-similarity', Indiana Univ. Math. J. 30 (1981), no. 5, 713-747. MR0625600 (82h:49026)

[8] M. Ionescu, 'Operator Algebras and Mauldin-Williams graphs', preprint OA/0401408.

[9] T. Kajiwara and Y. Watatani, ' $C^{*}$-algebras associated with self-similar sets', preprint $\mathrm{OA} / 0312481$.

[10] T. Kajiwara and Y. Watatani, 'KMS states on $C^{*}$-algebras associated with self-similar sets', preprint OA/0405514.

[11] J. Kigami, Analysis on Fractals, 2001, Cambridge University Press. MR 1840042 (2002c:28015)

[12] A. Kumjian, D. Pask and I. Raeburn, 'Cuntz-Krieger algebras of directed graphs', Pacific J. Math. 184 (1998), no. 1, 161-174. MR.1626528(99i:46049)

[13] E. C. Lance, Hilbert $C^{*}$-modules. A toolkit for operator algebraists, London Mathematical Society Lecture Note Series, 210. Cambridge University Press, Cambridge, 1995. MR 1325694 (96k:46100) 
[14] R. D. Mauldin and S. C. Williams, 'Hausdorff dimension in graph directed constructions', Trans. Amer. Math. Soc. 309 (1988), no. 2, 811-829. MR0961615(89i:28003)

[15] P. S. Muhly and B. Solel, 'Tensor algebras over $C^{*}$-correspondences: representations, dilations, and $C^{*}$-envelopes', J. Funct. Anal. 158 (1998), no. 2, 389-457. MR1648483 (99j:46066)

[16] P. S. Muhly and B. Solel, 'On the Morita equivalence of tensor algebras', Proc. London Math. Soc. (3) 81 (2000), no. 1, 113-168. MR.1757049 (2001g:46128)

[17] M. V. Pimsner, 'A class of $C^{*}$-algebras generalizing both Cuntz-Krieger algebras and crossed products by $\mathbb{Z}$ ', Free probability theory (Waterloo, ON, 1995), 189-212, Fields Inst. Commun., 12, Amer. Math. Soc., Providence, RI, 1997. MR1426840 (97k:46069)

[18] C. Pinzari, Y. Watatani and K. Yonetani, 'KMS states, entropy and the variational principle in full $C^{*}$-dynamical systems', Comm. Math. Phys. 213 (2000), no. 2, 331-379. MR.1785460 (2002a:46097)

[19] I. Raeburn, 'On the Picard group of a continuous trace $C^{*}$-algebra', Trans. Amer. Math. Soc. 263 (1981), no. 1, 183-205. MR0590419 (82b:46090)

[20] I. Raeburn and D. P. Williams, Morita equivalence and continuous-trace $C^{*}$-algebras, Mathematical Surveys and Monographs, 60. American Mathematical Society, Providence, RI, 1998. MR.1634408 (2000c:46108)

[21] M. A. Rieffel, 'Induced representations of $C^{*}$-algebras', Advances in Math. 13 (1974), 176257. MR0353003 (50:5489)

[22] M. A. Rieffel, 'Morita equivalence for $C^{*}$-algebras and $W^{*}$-algebras', J. Pure Appl. Algebra 5 (1974), 51-96. MR0367670(51:3912)

Department of Mathematics, The University of Iowa, Iowa City, Iowa 52242

E-mail address: mionescu@math.uiowa.edu 